\title{
Positive Pseudomonas Culture
}

National Cancer Institute

\section{Source}

National Cancer Institute. Positive Pseudomonas Culture. NCI Thesaurus. Code C122582.

A laboratory test result demonstrating the presence of bacteria from the genus Pseudomonas in a sample after culture. 